EPSC Abstracts

Vol. 15, EPSC2021-292, 2021

https://doi.org/10.5194/epsc2021-292

Europlanet Science Congress 2021

(C) Author(s) 2021. This work is distributed under

the Creative Commons Attribution 4.0 License.

\title{
Photometric behavior of the Ryugu's spectral parameters retrieved from NIRS3 spectra
}

\author{
Andrea Longobardo ${ }^{1}$, Ernesto Palomba ${ }^{1}$, Anna Galiano ${ }^{1}$, Fabrizio Dirri ${ }^{1}$, Angelo Zinzi ${ }^{2}$, Mario \\ D'Amore 3 , Deborah Domingue ${ }^{4}$, Kohei Kitazato ${ }^{5}$, Stefan Schroeder ${ }^{3}$, and the Hayabusa2/NIRS3* \\ ${ }^{1}$ IAPS-INAF, IAPS, Rome, Italy (andrea.longobardo@iaps.inaf.it) \\ ${ }^{2}$ ASI-SSDC, Rome, Italy \\ ${ }^{3}$ German Aerospace Center, Institute of Planetary Research, Berlin, Germany \\ ${ }^{4}$ PSI, Tucson, AZ, USA \\ ${ }^{5}$ University of Aizu, Aizu-Wakamatsu 965-8580, Fukushima, Japan \\ ${ }^{*} A$ full list of authors appears at the end of the abstract
}

\section{Introduction}

The JAXA/Hayabusa2 mission observed the Ryugu asteroid for one year and half (e.g., Yoshikawa, 2021) and acquired images by means of the ONC camera (Sugita et al., 2019) and NIR spectra in the 1.8-3.6 microns range thanks to the NIRS3 spectrometer (Iwata et al., 2017).

Ryugu is a very dark body (albedo of 0.043, Sugita et al., 2019) and its NIR spectrum is characterized by the shallow 2.72 and 2.8 microns absorption bands, ascribed to $\mathrm{OH}$ stretiching and thus indicating a weak hydration (Kitazato et al., 2019).

Photometric properties are very useful to infer optical and physical properties of the surface. The Ryugu photometry in the visible range has been studied by Tatsumi et al. (2020), which retrieved the average geometric albedo and the visible phase reddening. Pilorget et al. (2020) studied the NIR photometry observing a local anti-correlation between albedo and band depth.

In this work, we focus on the photometric behavior of the main spectral parameters, describing the Ryugu's NIR spectrum, i.e., infrared reflectance, albedo and spectral slope.

\section{Method}

We performed a statistical analysis of the NIRS3 dataset acquired between the two Hayabusa2 touchdown operations on Ryugu (i.e., between February and July 2019). The method is based on an empirical model already applied on several small bodies (e.g., Longobardo et al., 2017, 2019) and consists of the following steps:

- Removal of the topography influence by applying a disk function which removes the influence of incidence and emission angles. We selected the Akimov disk function (Shkuratov et al., 1999);

- Retrieval of the median value of spectral parameters at different phase angles; 
- Fit of the median spectral parameter as a function of the phase angle;

- Comparison with behaviors observed on other small bodies

\section{Results and discussion.}

Infrared albedo. Due to the small phase angle coverage $\left(15^{\circ}-40^{\circ}\right)$, we approximated the phase function as a straight line (Figure 1) and compared its slope with the slope of the phase function of other asteroids retrieved in the infrared range. We found a similarity between Ceres and Ryugu and flatter phase functions for Eros and Vesta, confirming the well known anti-correlation between albedo and phase function steepness.

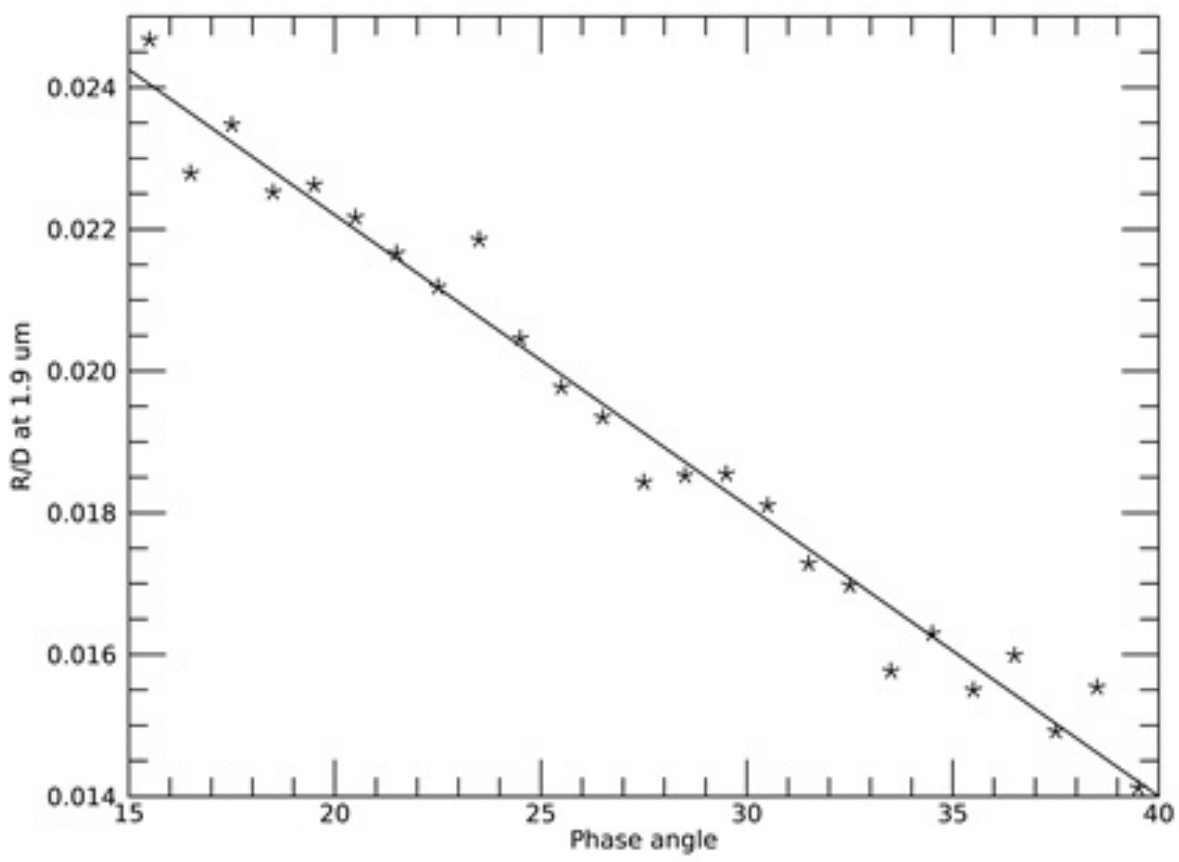

Figure 1. Ryugu's phase function.

2.72 and 2.8 band depths. Band depths are observed to decrease with increasing phase angle. This is a unique behavior that has not been observed on any other small body visited by a space mission. This behavior can be ascribed to Ryugu's very dark surface ( $2 \%$ of incident light is reflected from the surface, Sugita et al., 2019; Kitazato et al., 2019), in which the role of multiple scattering is negligible and the large absorption properties of the surface reduces the radiation reflected at larger phase angles.

Photometrically corrected band depth seems uncorrelated with albedo. This does not contradict the outcomes by Pilorget et al. (2020), which found a local anticorrelation. As a matter of fact, the anticorrelation is observed locally and limited to the darkest regions, while we studied a global behavior.

Infrared slope. A small phase reddening is observed, very similar to that observed by the ONC camera in the visible range (Tastumi et al., 2020). This is a different behavior with respect to other 
small bodies, where the visible phase reddening is generally larger than the infrared one. This could be ascribed to absence of multiple scattering and/or to microscopically smooth particles on Ryugu

Photometrically corrected infrared slope anticorrelated with albedo, confirming outcomes of previous works (e.g., Galiano et al., 2020).

\section{Conclusions}

The Ryugu's photometric behavior is generally similar to other dark asteroids. Nevertheless, the photometric behavior of spectral parameters is some cases is different, mainly due to its very low albedo and probably to smoother particles.

\section{References:}

Galiano et al., 2020, Icarus 351, 113959; Iwata et al., 2017, SSR 208, 317-337; Kitazato et al., 2019, Science 364, 6367, 272-275; Longobardo et al., 2017, MNRAS 469, 346-356; Longobardo et al., 2019, Icarus 240, 20-35; Pilorget et al., 2020, Icarus 355, 114-126; Sugita et al., 2019, Science 364, 6437, 252; Tatsumi et al., 2020, A\&A 639, A83; Yoshikawa, 2021, in Sample Return Missions, Elsevier, ISBN 9780128183304

Hayabusa2/NIRS3: T. Iwata, M. Matsuoka, T. Hiroi, D. Takir, T. Nakamura, M. Abe, M. Ohtake, S. Matsuura, S. Watanabe, M. Yoshikawa, T. Saiki, S. Tanaka, T. Okada, Y. Yamamoto, Y. Takei, K. Shirai, N. Hirata, N. Hirata, K. Matsumoto, Y. Tsuda 